\title{
The impact of preadmission oral bisphosphonate use on 30-day mortality following stroke: a population-based cohort study of I00,043 patients
}

This article was published in the following Dove Press journal:

Clinical Epidemiology

24 August 2015

Number of times this article has been viewed

\author{
Diana Hedevang \\ Christensen ${ }^{1,2}$ \\ Erzsébet Horváth-Puhó' \\ Morten Schmidt' \\ Christian Fynbo \\ Christiansen' \\ Lars Pedersen' \\ Bente Lomholt Langdahl ${ }^{2}$ \\ Reimar Wernich Thomsen' \\ 'Department of Clinical Epidemiology, \\ ${ }^{2}$ Department of Endocrinology and \\ Internal Medicine, Aarhus University \\ Hospital, Aarhus, Denmark
}

Correspondence: Diana Hedevang

Christensen

Department of Clinical Epidemiology,

Aarhus University Hospital, Olof

Palmes Allé 43-45, DK-8200,

Aarhus N, Denmark

Tel +45 25306121

Fax +45 8716 72I5

Email dhcr@clin.au.dk
Purpose: Bisphosphonate use has been associated with increased risk of fatal stroke. We examined the association between preadmission use of oral bisphosphonates and 30-day mortality following hospitalization for stroke.

Patients and methods: We conducted a nationwide population-based cohort study using medical databases and identified all patients in Denmark with a first-time hospitalization for stroke between 1 July 2004 and 31 December 2012 (N=100,043). Cox regression was used to compute adjusted hazard ratios as a measure of 30-day mortality rate ratios (MRRs) associated with bisphosphonate current use (prescription filled within 90 days prior to the stroke) or recent use (prescription filled in the 90-180 days prior to the stroke). Current use was further classified as new or long-term use.

Results: We found 51,982 patients with acute ischemic stroke (AIS), 11,779 with intracerebral hemorrhage (ICH), 4,528 with subarachnoid hemorrhage (SAH), and 31,754 with unspecified stroke. Absolute 30-day mortality risks were increased among current vs nonusers of bisphosphonates for AIS (11.9\% vs 8.5\%), ICH (43.2\% vs 34.5\%), SAH (40.3\% vs $23.2 \%$ ), and unspecified strokes (18.8\% vs 14.0\%). However, in adjusted analyses, current bisphosphonate use did not increase 30-day mortality from AIS (MRR, 0.87; 95\% confidence interval [CI]: 0.75, 1.01); ICH (MRR, 1.05; 95\% CI: 0.90, 1.23); SAH (MRR, 1.15; 95\% CI: 0.83, 1.61); or unspecified stroke (MRR, 0.94; 95\% CI: 0.81, 1.09). Likewise, no association with mortality was found for recent use. Adjusted analyses by type of bisphosphonate showed increased mortality following stroke among new users of etidronate (MRR, 1.40; 95\% CI: 1.01, 1.93) and reduced mortality after AIS among current users of alendronate (MRR, 0.87; 95\% CI: 0.74, 1.02).

Conclusion: We found no overall evidence that preadmission bisphosphonate use increases 30-day mortality following stroke.

Keywords: prognosis, oral bisphosphonates, stroke, mortality, cardiovascular disease, osteoporosis treatment

\section{Introduction}

Bisphosphonates are the first-line treatment for osteoporosis and are frequently prescribed in the elderly population. ${ }^{1}$ They inhibit osteoclast-mediated bone resorption and are effective in reducing bone loss and preventing fractures in osteoporotic patients and in patients receiving long-term glucocorticoid treatment. ${ }^{1}$ In 2007, a randomized controlled trial (RCT) showed an increased risk of cardiac arrhythmias and serious atrial fibrillation (AF) associated with the intravenously administered bisphosphonate, zoledronic acid. ${ }^{2}$ Since then, several studies have investigated the link between AF and use of bisphosphonates, with conflicting results. ${ }^{1,3,4}$

Because AF is a major risk factor for ischemic stroke, ${ }^{5}$ studies also have addressed whether bisphosphonates are associated with increased stroke risk. ${ }^{2,4,6-15}$ A Danish 
nationwide observational cohort study of 97.511 bisphosphonate users and 292.533 age and sex-matched controls found an increased risk of fatal stroke (death within 30 days of stroke) associated with increasing adherence to the treatment with etidronate and alendronate. ${ }^{11}$ The association with stroke incidence was less clear, indicating that bisphosphonates might worsen the prognosis of stroke. ${ }^{11}$ In the Health Outcomes and Reduced Incidence with Zoledronic Acid Once Yearly (HORIZON) Pivotal Fracture Trial, individuals exposed to zoledronic acid had a similar incidence of serious stroke-related adverse events than placebo users, but a nonsignificantly higher proportion of stroke-related deaths (87 events, 20 deaths from stroke) vs placebo group ( 88 events, eleven deaths from stroke), also pointing to worsened stroke prognosis. ${ }^{2}$ In contrast, other RCTs primarily investigating the effect of zoledronic acid on fracture risk and bone mineral density (BMD) found no association between bisphosphonate use and risk of fatal stroke. ${ }^{13,14}$

To our knowledge, no study has previously explored the impact of bisphosphonate use on subsequent mortality in a large cohort of stroke patients.

Since bisphosphonates are increasingly prescribed as the population ages, and stroke is a frequent disease among the elderly, ${ }^{16}$ any possible association between bisphosphonate use and worsened stroke prognosis is of clinical importance. We therefore conducted a nationwide population-based cohort study to examine the association of preadmission bisphosphonate use and 30-day mortality following hospitalization with stroke.

\section{Patients and methods}

\section{Setting}

We conducted this population-based cohort study in Denmark using information from three nationwide databases: the National Health Service Prescription Database (NHSPD) (previously named the Danish National Database of Reimbursed Prescriptions), ${ }^{17}$ the Danish Civil Registration System (CRS), ${ }^{18}$ and the Danish National Patient Registry (DNPR). ${ }^{19}$ Since 1968, all Danish residents have been assigned a unique Civil Personal Register number at birth or upon immigration, which permits accurate and unambiguous individual-level linkage across the databases. ${ }^{18}$

During the study period (July 1, 2004-December 31, 2012), the cumulative Danish population numbered 6,379,918 persons, including 3,207,231 women. The NHSPD contains complete data on all reimbursed drugs sold in Denmark since 1 January $2004,{ }^{17}$ and the study period was chosen to ensure availability of at least a 6-month prescription history for all study participants.
The Danish National Health Service provides all Danish residents with equal tax-supported access to health care. Services include access to general practitioners and hospitals and partial reimbursement for prescribed drugs. ${ }^{17}$

\section{Stroke}

We used the DNPR to identify all patients with a first-time inpatient primary or secondary diagnosis of acute ischemic stroke (AIS), intracerebral hemorrhage (ICH), subarachnoid hemorrhage $(\mathrm{SAH})$, or unspecified stroke during the study period. Despite the different underlying pathologies for these stroke subtypes, with AF being associated mainly with AIS, we choose to investigate all types of stroke because the study of Vestergaard et al ${ }^{11}$ included both AIS and ICH, and the HORIZON Pivotal Fracture Trial ${ }^{2}$ did not differentiate between stroke subtypes. Since its establishment in 1977 , the DNPR has collected data on admission and discharge dates from all Danish nonpsychiatric hospitals. ${ }^{19}$ Data on visits to emergency rooms and outpatient specialist clinics have been collected since $1995 .{ }^{19}$ Each hospital discharge is associated with one primary diagnosis and up to 19 secondary diagnoses, coded by physicians and classified according to the International Classification of Diseases, eighth revision (ICD-8) until the end of 1993 and the tenth revision (ICD-10) thereafter. ${ }^{19}$ In Denmark, $~ 90 \%$ of all patients with stroke are hospitalized and thus included in the DNPR. ${ }^{20}$

From the DNPR, we also obtained information on diagnostic procedures performed during hospitalization, ie, computed tomography (CT) and magnetic resonance imaging (MRI).

\section{Bisphosphonates}

We used the NHSPD to retrieve information on bisphosphonate prescriptions redeemed by stroke patients before their hospital admission date. The NHSPD contains information on reimbursable drugs dispensed at all pharmacies in Denmark. ${ }^{17}$ This registry includes the following information for each redeemed prescription: the patient's Civil Personal Register number, the amount and type of drug prescribed according to the Anatomical Therapeutic Chemical classification system, and the date on which the drug was dispensed. ${ }^{17}$

We defined current use as at least one recorded prescription for bisphosphonates within 90 days before the hospital admission for stroke. Because some adverse effects might be more frequent shortly after initiation of therapy, we also examined new use (defined as a first-ever prescription filled within 90 days before the hospital admission date). ${ }^{21}$ Long-term use was defined as a prescription redemption within 90 days before the admission date in combination 
with a minimum of one prescription redemption 90-180 days before admission and eventually additional prescriptions before this period. Recent use was defined as redemption of the most recent prescription between 90 days and 180 days before hospital admission. Nonuse was defined as no redeemed prescriptions within 180 days before hospital admission.

\section{Mortality}

We obtained information on all-cause mortality from the CRS, which has recorded all changes in vital status and migration for the entire Danish population since 1968, with daily electronic updates. ${ }^{18}$

\section{Potential confounders}

We searched the DNPR for patient characteristics that may be associated both with stroke prognosis and use of bisphosphonates, as recorded from 1977 up to the admission date. These included AF, myocardial infarction, intermittent arterial claudication, dementia, diabetes, angina pectoris, heart valve disease, venous thromboembolism, obesity, chronic kidney disease, hypertension, cancer, chronic pulmonary disease (COPD), and alcoholism-related disease. To obtain more complete information on excessive alcohol use and to increase the sensitivity of the diagnoses of diabetes mellitus and COPD, we retrieved data on prescriptions for alcohol deterrents, glucose-lowering medications, and respiratory medications from the prescription database ${ }^{17}$ and included these data in the respective variables. Severe osteoporosis and osteoporotic fractures, especially hip fractures are associated with increased mortality in general. ${ }^{22}$ From the DNPR, we retrieved each patient's hospital contact history of osteoporosis and/or osteoporotic fractures (hip fractures, vertebral fractures and distal forearm fractures) before the stroke and defined three categories: recent fracture (fracture within 6 months of stroke admission), nonrecent fracture (fracture $\geq 6$ months before stroke admission), and simple osteoporosis (without any fracture diagnosis).

From the DNPR, we also obtained information on current use (last prescription redemption within 90 days before admission) of hormone replacement therapy, nonsteroidal anti-inflammatory drugs, selective serotonin reuptake inhibitors, systemic glucocorticoids, vitamin $\mathrm{K}$ antagonists, clopidogrel, acetylsalicylic acid, statins, nitrates (if $\geq 2$ prescriptions), diuretics, calcium channel blockers, beta-blockers, angiotensin-converting enzyme inhibitors, and angiotensin-II receptor antagonists. Relevant ICD-codes and Anatomical Therapeutic Chemical codes are provided in Table S1. http://www.dovepress.com/get supplementary file. php?f=85427.pdf

\section{Statistical analysis}

We characterized the cohort according to sex, age group ( $<60$ years, 60-69 years, 70-79 years, or $\geq 80$ years), comorbidities, comedication use, and whether CT or MRI was performed during hospitalization. Patients were censored at death, at emigration, on December 31, 2012, or after 30 days of follow-up following the hospital admission date, whichever occurred first.

We computed absolute 30-day mortality risks using Kaplan-Meier method. We used a Cox proportional hazards regression model to compute hazard ratios (HRs) as a measure of mortality rate ratios (MRRs) within 30 days of hospital admission for current, recent, long, and new use of bisphosphonates compared with nonuse. We also examined alendronate, etidronate, risedronate, and ibandronate separately. To control for confounding, the model was adjusted for the potential confounders listed earlier. In a sensitivity analysis, we ran the model excluding AF and vitamin $\mathrm{K}$ antagonist use (marker of AF) as potential intermediate steps, to assess if any effect of bisphosphonates on stroke mortality was independent of AF and its treatment. We did an additional analysis of long-term users defining long-term use as minimum one prescription redemption 0-90 days prior to the stroke and minimum one additional prescription redemption 365 days to 2 years prior to the stroke.

The analyses were stratified by age group, sex, simple osteoporosis, recent and nonrecent osteoporotic fracture, and AF. We repeated the analyses restricted to patients with a CT or MRI scan registered in the DNPR during their hospitalization, in order to increase the positive predicted value of the stroke diagnosis. To examine the sensitivity of the estimates to differences in exposure definitions, we repeated the analysis using a 30-day, a 60-day, and a 180-day exposure window instead of a 90-day window. To reduce the potential for confounding by indication for bisphosphonate use, we also directly compared current users with recent users.

Finally, we conducted a propensity score-matched analysis. As previously described, ${ }^{23}$ we generated a logistic regression model that predicted bisphosphonate use among stroke patients conditional on the variables included in the multivariable model described earlier. ${ }^{24}$ We calculated the propensity score, ie, the probability of bisphosphonate use for each stroke patient. The propensity score distribution among users and nonusers was visually inspected. 
Each bisphosphonate user was matched with a nonuser with the closest propensity score by using a greedy matching algorithm. ${ }^{25}$ The matching was performed without replacement, separately for the individual types of bisphosphonates, and within a maximum matching range (caliper width) in propensity score of $\pm 0.025 .{ }^{25}$ We repeated the Cox regression model with robust standard errors to account for clustering in matched pairs, and compared mortality rates between bisphosphonate users and propensity score-matched nonusers. ${ }^{25}$

The proportional hazard assumption was visually assessed using $\log -\log$ plots and found valid.

All analyses were performed using SAS version 9.2 (SAS Institute Inc., Cary, NC, USA).

This registry-based study was approved by the Danish Data Protection Agency (record number 2011-41-5755). The study did not require further ethics approval as it did not involve patient contact or any intervention.

\section{Results}

Table 1 presents patient characteristics. The total study population included 100,043 patients with a first-time stroke between 2004 and 2012. Among these, 51.982 (52.0\%) patients had AIS (median age: 73 years), 11,779 (11.8\%) patients had ICH (median age: 72 years), 4,528 (4.5\%) patients had SAH (median age: 58 years), and 31.754 (31.7\%) patients had unspecified stroke (median age: 76 years). In general, stroke was more common among men, but for each type of stroke, women were overrepresented among bisphosphonate users. A total of 3,427 patients (3.4\%) were current bisphosphonates users, $652(0.7 \%)$ were recent users, and 95,964 (95.9\%) were nonusers. Among current bisphosphonates users, $82.3 \%$ used alendronate, $10.3 \%$ used etidronate, $5.7 \%$ used ibandronate, and $2.0 \%$ used risedronate (Table S2). Bisphosphonate users were older than nonusers and also had a higher prevalence of fractures, angina pectoris, AF, heart valve disease, hypertension, dementia, COPD, and cancer. Bisphosphonate users also more frequently had prescriptions for hormone replacement therapy, glucocorticoids, nonsteroidal anti-inflammatory drugs, selective serotonin reuptake inhibitors, and most cardiovascular drugs.

Table 2 presents mortality data. Absolute 30-day mortality risks were increased among current vs nonusers of bisphosphonates for AIS (11.9\% vs 8.5\%), ICH (43.2\% vs $34.5 \%)$, SAH (40.3\% vs $23.2 \%$ ), and unspecified stroke (18.8\% vs $14.0 \%$ ), and this was reflected in the unadjusted analyses in which current use of bisphosphonates, compared with nonuse, showed increased 30-day mortality for AIS $(\mathrm{MRR}=1.43,95 \%$ confidence interval $[\mathrm{CI}]: 1.24,1.64)$,
ICH $(\mathrm{MRR}=1.37,95 \% \mathrm{CI}: 1.19,1.58), \mathrm{SAH}(\mathrm{MRR}=1.82$, $95 \%$ CI: $1.35,2.45)$, and unspecified stroke $(\mathrm{MRR}=1.39$, 95\% CI: 1.21, 1.59). However, after adjustment for potential confounders, current use of bisphosphonates showed no association with mortality following ICH $(\mathrm{MRR}=1.05$, 95\% CI: 0.90, 1.23), SAH (MRR $=1.15,95 \%$ CI: 0.83, 1.61), or unspecified stroke $(\mathrm{MRR}=0.94,95 \% \mathrm{CI}: 0.81,1.09)$, whereas for AIS, a tendency toward reduced 30-day stroke mortality was observed $(\mathrm{MRR}=0.87,95 \% \mathrm{CI}: 0.75,1.01)$. Recent use of bisphosphonates showed no impact on 30-day mortality. The estimates from the propensity score-matched analyses tended to be a little higher than the conventionally adjusted estimates, but in general, the results from the two methods were in agreement. Subanalyses according to the type of bisphosphonate showed an increased adjusted MRR for new users of etidronate (Table S3): Stroke overall $=1.40$ (95\% CI: $1.01 ; 1.93), \mathrm{ICH}=1.52$ (95\% CI: 0.81, 2.84), and unspecified stroke $=1.76$ (95\% CI: 1.09, 2.84), which was confirmed in the propensity score-matched analyses: stroke overall $=1.50$ (95\% CI: 1.03, 2.17), ICH =1.82 (95\% CI: 0.88 , 3.76), and unspecified stroke $=1.74$ (95\% CI: 1.00, 3.04). A subanalysis for alendronate revealed reduced MRR for AIS in conventionally adjusted analyses (MRR current $=0.87$, 95\% CI: $0.74,1.02$ ) and in propensity-score-matched analyses $($ MRR current $=0.90,95 \%$ CI: 0.74, 1.10) $($ Table S4). For the remaining types of bisphosphonates, none of the separate analyses by drug type had any impact on 30-day mortality (Tables S5 and S6).

MRR estimates were not changed materially when comparing current users with recent users, restricting the analysis to CT- or MRI-confirmed cases, when using a 30-/60-/180-day exposure window, or when extending the definition of long-term use (data not shown). No notable effect modification was observed within strata of age, sex, AF, recent fracture, nonrecent fracture or simple osteoporosis (Table S7). Excluding AF and vitamin $\mathrm{K}$ antagonists from the multivariate model did not materially affect the estimates; eg, MRRs with current bisphosphonate use: AIS $=0.86(95 \%$ CI: $0.74,1.00), \mathrm{ICH}=1.06$ (95\% CI: 0.91, 1.24), $\mathrm{SAH}=1.16$ (95\% CI: $0.83,1.62)$, and unspecified stroke $=0.94(95 \% \mathrm{CI}$ : $0.82,1.09$ ) (Table S8).

\section{Discussion}

In our study, preadmission bisphosphonate use reassuringly showed no increased 30-day mortality following a first-time hospitalization for AIS, ICH, SAH, or unspecified stroke. This finding was consistent in separate analyses of alendronate, ibandronate, and risedronate. For etidronate, 


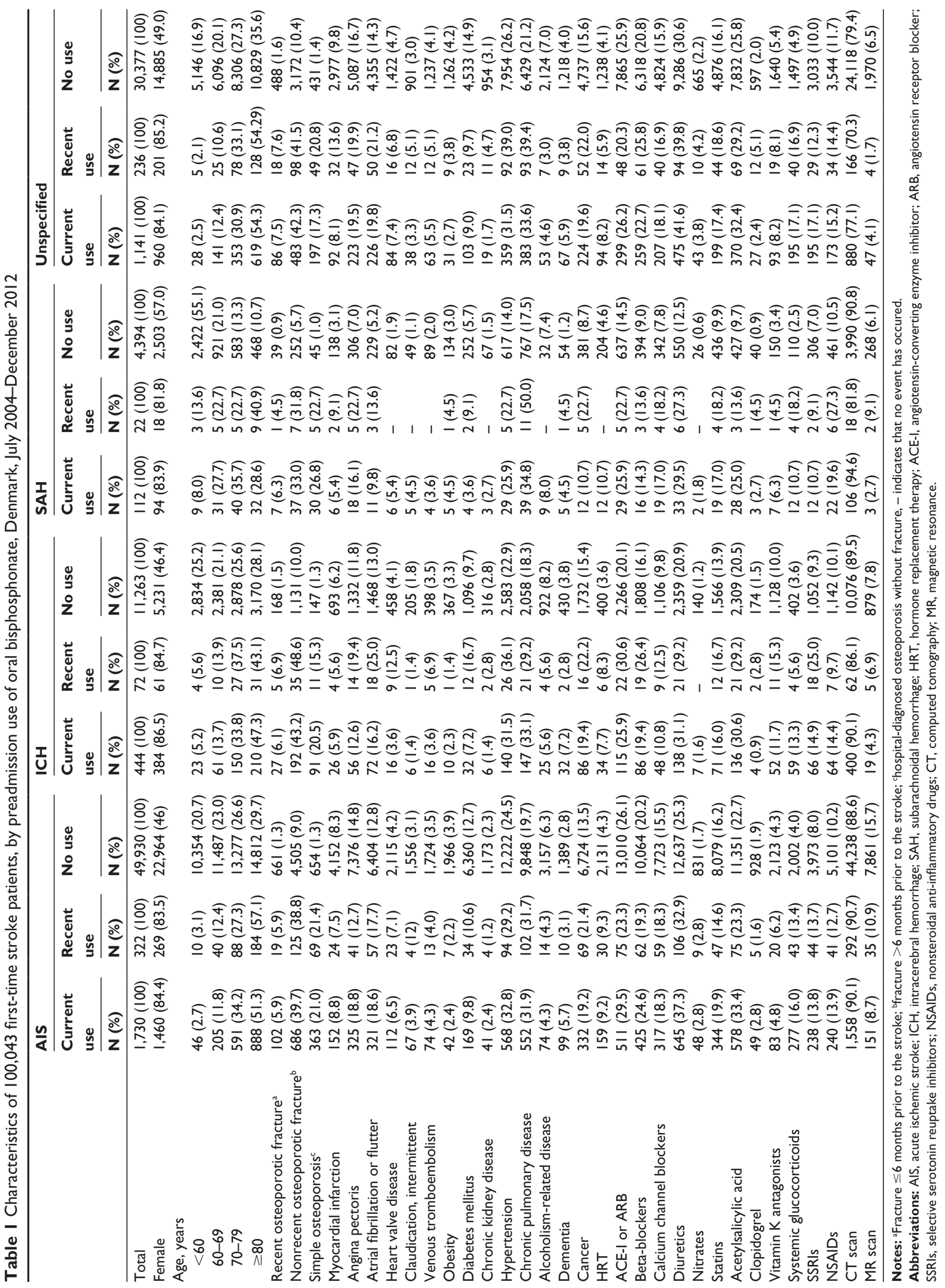


Table 2 Preadmission bisphosphonate use and 30-day mortality risk and mortality rate ratios following stroke

\begin{tabular}{|c|c|c|c|c|}
\hline & Mortality risk \% $(95 \% \mathrm{CI})$ & MRRs $(95 \% \mathrm{Cl})$ unadjusted & MRRs $(95 \% \mathrm{Cl})$ adjusted $^{a}$ & PS-matched $(95 \% \mathrm{Cl})$ \\
\hline \multicolumn{5}{|l|}{ Stroke overall } \\
\hline No use $e^{b}$ & $13.9(|3.7 ;| 4.2)$ & I (reference) & I (reference) & I (reference) \\
\hline Current use ${ }^{c}$ & $19.2(17.9 ; 20.5)$ & $1.42(1.31 ; 1.53)$ & $0.97(0.89 ; 1.05)$ & $0.94(0.85 ; 1.05)$ \\
\hline Recent use $^{d}$ & $20.2(I 7.3 ; 23.5)$ & $1.49(1.26 ; 1.77)$ & $0.99(0.83 ; 1.18)$ & $1.12(0.87 ; 1.43)$ \\
\hline New use $e^{e}$ & $20.8(18.0 ; 23.9)$ & $1.55(1.32 ; 1.83)$ & $1.06(0.90 ; 1.25)$ & I.II (0.88; I.40) \\
\hline Long-term use $^{f}$ & $18.7(17.3 ; 20.3)$ & $1.38(1.26 ; 1.51)$ & $0.94(0.85 ; 1.03)$ & $0.98(0.86 ; 1.10)$ \\
\hline \multicolumn{5}{|l|}{ AIS } \\
\hline No use & $8.5(8.2 ; 8.7)$ & I (reference) & I (reference) & I (reference) \\
\hline Current use & $11.9(10.4 ; 13.5)$ & $1.43(1.24 ; 1.64)$ & $0.87(0.75 ; 1.01)$ & $0.90(0.74 ; 1.08)$ \\
\hline Recent use & 14.7 (II.2; 19.0) & $1.80(1.35 ; 2.40)$ & I.I2(0.84; I.50) & $1.25(0.82 ; 1.91)$ \\
\hline New use & $11.5(8.6 ; 15.4)$ & $1.39(1.02 ; 1.89)$ & $0.80(0.58 ; 1.10)$ & $0.97(0.63 ; 1.50)$ \\
\hline Long-term use & $12.0(10.4 ; 13.8)$ & $1.44(1.23 ; 1.68)$ & $0.88(0.75 ; 1.04)$ & $1.06(0.85 ; 1.33)$ \\
\hline \multicolumn{5}{|l|}{$\mathrm{ICH}$} \\
\hline No use & $34.5(33.7 ; 35.4)$ & I (reference) & I (reference) & I (reference) \\
\hline Current use & $43.2(38.8 ; 48.0)$ & 1.37 (1.19; 1.58) & $1.05(0.90 ; 1.23)$ & $1.02(0.83 ; 1.24)$ \\
\hline Recent use & $43.4(32.8 ; 55.6)$ & $1.37(0.96 ; 1.95)$ & $1.03(0.72 ; 1.47)$ & $0.98(0.60 ; 1.61)$ \\
\hline New use & $44.3(35.1 ; 54.8)$ & $1.42(1.05 ; 1.92)$ & $1.07(0.79 ; 1.46)$ & $1.29(0.83 ; 2.00)$ \\
\hline Long-term use & $42.9(37.9 ; 48.3)$ & $1.36(1.15 ; 1.60)$ & $1.04(0.88 ; 1.24)$ & $0.99(0.79 ; 1.24)$ \\
\hline \multicolumn{5}{|l|}{$\mathrm{SAH}$} \\
\hline No use & $23.2(22.0 ; 24.5)$ & I (reference) & I (reference) & I (reference) \\
\hline Current use & $40.3(31.9 ; 50.0)$ & I.82 (1.35; 2.45) & $1.15(0.83 ; 1.61)$ & I. $.44(0.92 ; 2.26)$ \\
\hline Recent use & $31.8(16.6 ; 55.4)$ & I.5I (0.72; 3.17) & $0.97(0.46 ; 2.08)$ & $\mathrm{I} .49(0.48 ; 4.6 \mathrm{I})$ \\
\hline New use & $50.0(32.2 ; 70.9)$ & $2.38(1.35 ; 4.20)$ & $1.52(0.85 ; 2.74)$ & $1.64(0.68 ; 3.97)$ \\
\hline Long-term use & $37.6(28.4 ; 48.7)$ & $1.67(1.18 ; 2.37)$ & I.05 (0.7I; I.54) & $1.27(0.76 ; 2.13)$ \\
\hline \multicolumn{5}{|l|}{ Unspecified stroke } \\
\hline No use & $14.0(13.6 ; 14.4)$ & I (reference) & I (reference) & I (reference) \\
\hline Current use & $18.8(16.6 ; 21.2)$ & 1.39 (1.21; 1.59) & $0.94(0.81 ; 1.09)$ & $0.88(0.73 ; 1.06)$ \\
\hline Recent use & $19.5(15.0 ; 25.2)$ & $1.42(1.06 ; 1.90)$ & $0.91(0.68 ; 1.22)$ & $1.04(0.69 ; 1.58)$ \\
\hline New use & $21.7(I 7.1 ; 27.4)$ & $1.63(1.25 ; 2.13)$ & $1.22(0.93 ; 1.59)$ & $1.09(0.75 ; 1.60)$ \\
\hline Long-term use & $17.9(15.6 ; 20.6)$ & 1.32 (1.12; 1.54) & $0.86(0.73 ; 1.02)$ & $0.86(0.70 ; 1.07)$ \\
\hline
\end{tabular}

Notes: adjusted for sex, age groups, recent osteoporotic fracture ( $\leq 6$ months), nonrecent osteoporotic fracture ( $>6$ months), simple osteoporosis (no fracture), myocardial infarction, angina pectoris, atrial fibrillation or flutter, heart valve disease, intermittent arterial claudication, venous thromboembolism, obesity, diabetes, chronic kidney disease, hypertension, chronic obstructive pulmonary disease (includes inhalation medications for chronic pulmonary obstructive disease), alcoholism-related diseases, dementia, cancer, and use of cardiovascular drugs (angiotensin-converting enzyme inhibitors, angiotensin-ll receptor blockers, beta-blockers, calcium channel blockers, diuretics, nitrates, statins, acetylsalicylic acid, clopidogrel, and vitamin $\mathrm{K}$ antagonists), hormone replacement therapy, systemic glucocorticoids, nonsteroidal anti-inflammatory drugs, and selective serotonin receptor inhibitors; ${ }^{b}$ no prescription redemption within $0-180$ days before admission date; ${ }^{c}$ prescription redemption within 90 days before

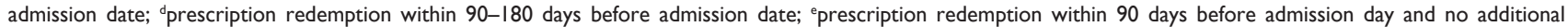
prescriptions; ' prescription redemption within 90 days before admission date and at least one additional prescription $90-180$ days prior to admission and eventually additional prescriptions before this period.

Abbreviations: MRRs, mortality rate ratios; $\mathrm{Cl}$, confidence interval; PS-matched, propensity-score-matched; AIS, acute ischemic stroke; ICH, intracerebral hemorrhage; SAH, subarachnoid hemorrhage.

we noted a possibly increased 30-day mortality following stroke associated with new use, but no association with current or recent use. For AIS, a slightly reduced short-term mortality, driven by alendronate, was seen.

The overall 30-day mortality for AIS ( 10\%), ICH $(\sim 40 \%)$, and SAH $(\sim 25 \%)$ in our study is comparable to previous studies of stroke prognosis in the general population, finding absolute 30-day mortality of $6.8 \%-12.6 \%$ for AIS, ${ }^{26,27} 13.1 \%-55.8 \%$ (median value $40.4 \%$ ) for $\mathrm{ICH},{ }^{28}$ and $25 \%-40 \%$ for $\mathrm{SAH}^{29}$

Previously, only a few studies have investigated bisphosphonate use and risk of fatal stroke - an endpoint that combines both stroke risk and prognosis. Of these, only one cohort study investigated oral bisphosphonates, ${ }^{11}$ finding an increased risk of fatal stroke associated with alendronate ( $\mathrm{HR}=2.05,95 \% \mathrm{CI}: 1.63,2.58)$ and etidronate ( $\mathrm{HR}=1.56,95 \% \mathrm{CI}: 1.35,1.80)$. Our data indicated worsened stroke prognosis, for ICH and unspecified stroke, solely for the small subgroup of new etidronate users, whereas in contrast to the previous study, we found no increase yet even a slight decrease in stroke mortality associated with alendronate use. This finding for alendronate could, in theory, be due to the nitrogen-containing bisphosphonates exerting arterial vascular protection through a cholesterol-lowering effect. ${ }^{30,31}$ However, in clinical studies, predominantly the nonnitrogen-containing bisphosphonate etidronate has been associated with reduced arterial vascular calcification and arteriosclerosis. ${ }^{32-36}$ 
It remains unclear to which degree these subgroup findings in our study may be due to chance. For etidronate, we speculate that the finding in new users may be related to potential protopathic bias, ie, early warning or prodromal symptoms of an impending stroke or other deteriorating health status predicting both etidronate initiation and subsequent stroke.

The more stroke-related deaths with zoledronic acid vs placebo in the HORIZON Pivotal Fracture Trial ${ }^{2}$ might be due to type 1 error. There is no firm evidence of any strong biological mechanisms that would readily explain any association of bisphosphonates and increased stroke mortality. Unfortunately, we lacked information of stroke severity, which is an important predictor of stroke outcome. ${ }^{26,27}$ Theoretically, the underlying mechanisms for an association between bisphosphonate use and stroke mortality could include an ability of - in particular - recently intravenously administered bisphosphonates to induce an acute phase response with release of proinflammatory cytokines including TNF- $\alpha,{ }^{30}$ which appear to exacerbate neuronal injury in ischemic stroke patients. ${ }^{37}$ Similar immune-modulating effects could in theory worsen the outcome of poststroke infections and thereby increase stroke mortality. ${ }^{30,38}$ Bisphosphonates might induce arrhythmias including AF, which is associated with more severe AIS $^{39}$ and is a strong adverse prognostic factor in first-time AIS. ${ }^{26,39,40}$ For ICH and $\mathrm{SAH}$, any effect on stroke prognosis might be indirect caused by anticoagulant therapy for AF. Nonetheless, the association of bisphosphonates with AF remains uncertain, and adjustment for known $\mathrm{AF}$ and vitamin $\mathrm{K}$ antagonist treatment did not materially affect the estimates in our study. Finally, bisphosphonates have been linked with increased risk of gastrointestinal bleeding. ${ }^{41}$ Such bleeding occurs only in $1.5 \%-7.8 \%$ of stroke events ${ }^{42-44}$ and is associated with increased mortality. ${ }^{44}$ Conclusively, a strong biological rationale behind bisphosphonates being associated with worsened stroke prognosis is lacking, which is in agreement with our null result. Moreover, other previous RCTs did not detect an increased risk of fatal stroke associated with use of zoledronic acid. ${ }^{13,14}$

Major strengths of our study include its nationwide population-based design, its large sample size, complete follow-up for all patients, and uniform data collection within the setting of a tax-supported universal health care system with independently and prospectively recorded hospital and prescription history. This setting reduced the biases often seen in observational studies based on selected patients or clinics.
Data on reimbursed prescriptions are nearly complete in the NHSPD. ${ }^{17}$ Since oral bisphosphonates are not sold over-the-counter in Denmark, we were able to identify all bisphosphonate users. However, compliance with bisphosphonate therapy is known to vary ${ }^{45}$ and potentially could have biased our results toward the null.

Our mortality data were virtually complete. ${ }^{18}$ The positive predictive value of acute stroke diagnoses in the DNPR is $97 \%$ for AIS, $74 \%$ for $\mathrm{ICH}$, and $67 \%$ for $\mathrm{SAH} .{ }^{46}$ In our study, $32 \%$ of patients had a diagnosis of unspecified stroke. A previous validation study found that two-thirds of unspecified strokes in DNPR were ischemic strokes. ${ }^{46}$

Adjustment for a range of covariates, including increased age, osteoporotic fractures, and other comorbidities, virtually eliminated the association between bisphosphonate use and increased stroke mortality that was observed in unadjusted analyses. As in any observational study, we cannot exclude residual confounding. We lacked information on low BMD, which is the main indication for bisphosphonate use. Low BMD is a risk factor for stroke, all-cause and cardiovascular death, while it does not seem to be associated with increased stroke mortality. ${ }^{47}$ If low BMD per se caused increased stroke mortality, ie, confounding by indication, this would have biased mortality estimates associated with bisphosphonate use upward, not changing our conclusion. In addition, our adjustment for hospital diagnosed osteoporosis and osteoporotic fractures likely reduced confounding by indication. On the other hand, a more comprehensive registration of potential confounders such as osteoporosis and comorbidities among bisphosphonate users vs nonusers may have led us to underestimate mortality associated with bisphosphonates. We speculate that, eg, the tendency toward reduced adjusted AIS mortality in bisphosphonate users may be due to such a more comprehensive registration of potential confounders. Also, compliant use of preventive medications like bisphosphonates may be associated with unmeasured healthy-user effects, which may also have caused underestimation of mortality associated with bisphosphonate use. However, we were able to adjust for other preadmission preventive medications like statins and antihypertensives, and we also to some degree adjusted for lifestyle factors through surrogate measures such as obesity, COPD, alcoholism-related disease, and cardiovascular diseases. Finally, use of vitamin D and calcium is likely more prevalent in people with low BMD than in healthy people. These drugs were not included in our adjusted model, since they are sold mostly over-the-counter in Denmark. The cardiovascular profile of vitamin D and calcium is unclear, ${ }^{48-50}$ with vitamin $\mathrm{D}$ deficiency being 
prevalent among patients admitted for stroke ${ }^{51}$ and low vitamin D level being inversely related to stroke severity and mortality, ${ }^{52,53}$ whereas calcium supplementation may increase cardiovascular risk. ${ }^{50}$

Generally, bisphosphonates are well tolerated. Our study provides evidence that bisphosphonates are safe to use with respect to severe cardiovascular disease. In conclusion, preadmission oral bisphosphonates did not impact 30-day mortality after hospitalization for stroke.

\section{Acknowledgments}

The study was supported by the Clinical Epidemiology Research Foundation and the Memorial Foundation of machinery manufacturer Jochum Jensen and wife Mette Marie Jensen, née Poulsen. None of the funding sources had a role in the design, conduct, analysis, or reporting of the study.

\section{Disclosure}

BLL is a member of the advisory boards for Merck (MSD), Amgen, Lilly, and UCB. The other authors report no personal conflicts of interest in this work. The Department of Clinical Epidemiology and the Department of Endocrinology and Internal Medicine, Aarhus University Hospital, receive funding for other studies from companies in the form of research grants to (and administered by) Aarhus University. None of these studies have any relation to the present study.

\section{References}

1. Suresh E, Pazianas M, Abrahamsen B. Safety issues with bisphosphonate therapy for osteoporosis. Rheumatology (Oxford). 2014;53(1):19-31.

2. Black DM, Delmas PD, Eastell R, et al; HORIZON Pivotal Fracture Trial. Once-yearly zoledronic acid for treatment of postmenopausal osteoporosis. N Engl J Med. 2007;356(18):1809-1822.

3. Howard PA, Barnes BJ, Vacek JL, Chen W, Lai SM. Impact of bisphosphonates on the risk of atrial fibrillation. Am J Cardiovasc Drugs. 2010; 10(6):359-367.

4. Sharma A, Chatterjee S, Arbab-Zadeh A, et al. Risk of serious atrial fibrillation and stroke with use of bisphosphonates: evidence from a meta-analysis. Chest. 2013;144(4):1311-1322.

5. Frost L, Engholm G, Johnsen S, Moller H, Husted S. Incident stroke after discharge from the hospital with a diagnosis of atrial fibrillation. Am J Med. 2000;108(1):36-40.

6. Abrahamsen B, Eiken P, Brixen K. Atrial fibrillation in fracture patients treated with oral bisphosphonates. J Intern Med. 2009;265(5): 581-592.

7. Christensen S, Mehnert F, Chapurlat RD, Baron JA, Sorensen HT. Oral bisphosphonates and risk of ischemic stroke: a case-control study. Osteoporos Int. 2011;22(6):1773-1779.

8. Wilkinson GS, Baillargeon J, Kuo YF, Freeman JL, Goodwin JS. Atrial fibrillation and stroke associated with intravenous bisphosphonate therapy in older patients with cancer. J Clin Oncol. 2010;28(33): 4898-4905.

9. Lu PY, Hsieh CF, Tsai YW, Huang WF. Alendronate and raloxifene use related to cardiovascular diseases: differentiation by different dosing regimens of alendronate. Clin Ther. 2011;33(9):1173-1179.
10. Kang JH, Keller JJ, Lin HC. A population-based 2-year follow-up study on the relationship between bisphosphonates and the risk of stroke. Osteoporos Int. 2012;23(10):2551-2557.

11. Vestergaard P, Schwartz K, Pinholt EM, Rejnmark L, Mosekilde L. Stroke in relation to use of raloxifene and other drugs against osteoporosis. Osteoporos Int. 2011;22(4):1037-1045.

12. Loke YK, Jeevanantham V, Singh S. Bisphosphonates and atrial fibrillation: systematic review and meta-analysis. Drug Saf. 2009;32(3): 219-228.

13. Black DM, Reid IR, Boonen S, et al. The effect of 3 versus 6 years of zoledronic acid treatment of osteoporosis: a randomized extension to the HORIZON-Pivotal Fracture Trial (PFT). J Bone Miner Res. 2012;27(2): 243-254.

14. Lyles KW, Colon-Emeric CS, Magaziner JS, et al; HORIZON Recurrent Fracture Trial. Zoledronic acid and clinical fractures and mortality after hip fracture. N Engl J Med. 2007;357(18):1799-1809.

15. Karam R, Camm J, McClung M. Yearly zoledronic acid in postmenopausal osteoporosis. NEngl J Med. 2007;357(7):712-713. [author reply 714-715].

16. Mukherjee D, Patil CG. Epidemiology and the global burden of stroke. World Neurosurg. 2011;76(6 Suppl):S85-S90.

17. Johannesdottir SA, Horvath-Puho E, Ehrenstein V, Schmidt M, Pedersen L, Sorensen HT. Existing data sources for clinical epidemiology: the Danish national database of reimbursed prescriptions. Clin Epidemiol. 2012;4:303-313.

18. Schmidt M, Pedersen L, Sorensen HT. The Danish civil registration system as a tool in epidemiology. Eur J Epidemiol. 2014;29(8): 541-549.

19. Lynge E, Sandegaard JL, Rebolj M. The Danish national patient register. Scand J Public Health. 2011;39(7 Suppl):30-33.

20. Schmidt M, Jacobsen JB, Johnsen SP, Botker HE, Sorensen HT. Eighteen-year trends in stroke mortality and the prognostic influence of comorbidity. Neurology. 2014;82(4):340-350.

21. Ray WA. Evaluating medication effects outside of clinical trials: newuser designs. Am J Epidemiol. 2003;158(9):915-920.

22. Abrahamsen B, van Staa T, Ariely R, Olson M, Cooper C. Excess mortality following hip fracture: a systematic epidemiological review. Osteoporos Int. 2009;20(10):1633-1650.

23. Schmidt M, Hovath-Puho E, Christiansen CF, Petersen KL, Botker HE, Sorensen HT. Preadmission use of nonaspirin nonsteroidal antiinflammatory drugs and 30-day stroke mortality. Neurology. 2014; 83(22):2013-2022.

24. Brookhart MA, Schneeweiss S, Rothman KJ, Glynn RJ, Avorn J, Sturmer T. Variable selection for propensity score models. Am J Epidemiol. 2006;163(12):1149-1156.

25. Austin PC. Propensity-score matching in the cardiovascular surgery literature from 2004 to 2006: a systematic review and suggestions for improvement. J Thorac Cardiovasc Surg. 2007;134(5):1128-1135.

26. Andersen KK, Andersen ZJ, Olsen TS. Predictors of early and late casefatality in a nationwide Danish study of 26,818 patients with first-ever ischemic stroke. Stroke. 2011;42(10):2806-2812.

27. Saposnik G, Hill MD, O'Donnell M, et al; Registry of the Canadian Stroke Network for the Stroke Outcome Research Canada (SORCan) Working Group. Variables associated with 7-day, 30-day, and 1-year fatality after ischemic stroke. Stroke. 2008;39(8):2318-2324.

28. van Asch CJ, Luitse MJ, Rinkel GJ, van der Tweel I, Algra A, Klijn CJ. Incidence, case fatality, and functional outcome of intracerebral haemorrhage over time, according to age, sex, and ethnic origin: a systematic review and meta-analysis. Lancet Neurol. 2010;9(2):167-176.

29. Lovelock CE, Rinkel GJ, Rothwell PM. Time trends in outcome of subarachnoid hemorrhage: population-based study and systematic review. Neurology. 2010;74(19):1494-1501.

30. Thompson K, Roelofs AJ, Jauhiainen M, Monkkonen H, Monkkonen J, Rogers MJ. Activation of gammadelta T cells by bisphosphonates. $A d v$ Exp Med Biol. 2010;658:11-20.

31. Ylitalo R. Bisphosphonates and atherosclerosis. Gen Pharmacol. 2000; 35(6):287-296. 
32. Ariyoshi T, Eishi K, Sakamoto I, Matsukuma S, Odate T. Effect of etidronic acid on arterial calcification in dialysis patients. Clin Drug Investig. 2006;26(4):215-222.

33. Kawahara T, Nishikawa M, Kawahara C, Inazu T, Sakai K, Suzuki G. Atorvastatin, etidronate, or both in patients at high risk for atherosclerotic aortic plaques: a randomized, controlled trial. Circulation. 2013; 127(23):2327-2335.

34. Koshiyama H, Nakamura Y, Tanaka S, Minamikawa J. Decrease in carotid intima-media thickness after 1-year therapy with etidronate for osteopenia associated with type 2 diabetes. J Clin Endocrinol Metab. 2000;85(8):2793-2796.

35. Nitta K, Akiba T, Suzuki K, et al. Effects of cyclic intermittent etidronate therapy on coronary artery calcification in patients receiving long-term hemodialysis. Am J Kidney Dis. 2004;44(4):680-688.

36. Tanko LB, Qin G, Alexandersen P, Bagger YZ, Christiansen C. Effective doses of ibandronate do not influence the 3-year progression of aortic calcification in elderly osteoporotic women. Osteoporos Int. 2005;16(2):184-190.

37. Lakhan SE, Kirchgessner A, Hofer M. Inflammatory mechanisms in ischemic stroke: therapeutic approaches. J Transl Med. 2009;7:97.

38. Chaudhry H, Zhou J, Zhong Y, et al. Role of cytokines as a double-edged sword in sepsis. In Vivo. 2013;27(6):669-684.

39. Appelros P, Nydevik I, Seiger A, Terent A. Predictors of severe stroke: influence of preexisting dementia and cardiac disorders. Stroke. 2002;33(10):2357-2362.

40. Vernino S, Brown RD Jr, Sejvar JJ, Sicks JD, Petty GW, O’Fallon WM. Cause-specific mortality after first cerebral infarction: a populationbased study. Stroke. 2003;34(8):1828-1832.

41. Peng YL, Hu HY, Luo JC, Hou MC, Lin HC, Lee FY. Alendronate, a bisphosphonate, increased upper and lower gastrointestinal bleeding: risk factor analysis from a nationwide population-based study. Osteoporos Int. 2014;25(5):1617-1623.

42. Davenport RJ, Dennis MS, Warlow CP. Gastrointestinal hemorrhage after acute stroke. Stroke. 1996;27(3):421-424.
43. Hsu HL, Lin YH, Huang YC, et al. Gastrointestinal hemorrhage after acute ischemic stroke and its risk factors in Asians. Eur Neurol. 2009;62(4):212-218.

44. O’Donnell MJ, Kapral MK, Fang J, et al; Investigators of the Registry of the Canadian Stroke Network. Gastrointestinal bleeding after acute ischemic stroke. Neurology. 2008;71(9):650-655.

45. Cramer JA, Gold DT, Silverman SL, Lewiecki EM. A systematic review of persistence and compliance with bisphosphonates for osteoporosis. Osteoporos Int. 2007;18(8):1023-1031.

46. Krarup LH, Boysen G, Janjua H, Prescott E, Truelsen T. Validity of stroke diagnoses in a national register of patients. Neuroepidemiology. 2007;28(3):150-154.

47. Qu X, Huang X, Jin F, et al. Bone mineral density and all-cause, cardiovascular and stroke mortality: a meta-analysis of prospective cohort studies. Int J Cardiol. 2013;166(2):385-393.

48. Wang L, Manson JE, Sesso HD. Calcium intake and risk of cardiovascular disease: a review of prospective studies and randomized clinical trials. Am J Cardiovasc Drugs. 2012;12(2):105-116.

49. Body JJ, Bergmann P, Boonen S, et al. Extraskeletal benefits and risks of calcium, vitamin D and anti-osteoporosis medications. Osteoporos Int. 2012;23(Suppl 1):S1-S23.

50. Bolland MJ, Grey A, Reid IR. Calcium supplements and cardiovascular risk: 5 years on. Ther Adv Drug Saf. 2013;4(5):199-210.

51. Poole KE, Loveridge N, Barker PJ, et al. Reduced vitamin D in acute stroke. Stroke. 2006;37(1):243-245.

52. Tu WJ, Zhao SJ, Xu DJ, Chen H. Serum 25-hydroxyvitamin D predicts the short-term outcomes of Chinese patients with acute ischaemic stroke. Clin Sci (Lond). 2014;126(5):339-346.

53. Daubail B, Jacquin A, Guilland JC, et al. Serum 25-hydroxyvitamin D predicts severity and prognosis in stroke patients. Eur J Neurol. 2013; 20(1):57-61.
Clinical Epidemiology

\section{Publish your work in this journal}

Clinical Epidemiology is an international, peer-reviewed, open access, online journal focusing on disease and drug epidemiology, identification of risk factors and screening procedures to develop optimal preventative initiatives and programs. Specific topics include: diagnosis, prognosis, treatment, screening, prevention, risk factor modification,

\section{Dovepress}

systematic reviews, risk \& safety of medical interventions, epidemiology \& biostatistical methods, and evaluation of guidelines, translational medicine, health policies \& economic evaluations. The manuscript management system is completely online and includes a very quick and fair peer-review system, which is all easy to use. 